\title{
A FUNCTIONAL CHARACTERIZATION OF PRIMITIVE BASE
}

\author{
HOWARD H. WICKE
}

\begin{abstract}
All published formulations of the concept of primitive base involve the concept of well ordering in a prominent way. This paper presents various conditions, not involving well ordering, on a function $h$ : $N \times X \rightarrow \tau$ such that if a space $(X, \tau)$ has a function satisfying one of these conditions, then it may be proved (using the axiom of choice) that it has a primitive base. These conditions are used in some characterizations of base of countable order. Examples show nonequivalence of the conditions with primitive base if the axiom of choice does not hold.
\end{abstract}

1. Introduction. The concept of primitive base is a simple and attractive one that is fundamentally involved in some important characterization theorems in the theory of generalized metric spaces [1], [7], [9]. Spaces with primitive bases bear a relation to spaces having base of countable order analogous to the relationship of quasi-developable spaces to developable spaces; primitive bases are the monotonic counterparts of quasi-developable spaces.

These bases have been characterized in several ways [8]. All of the formulations have in common the presence of a sequence of well-ordered open covers. The simplest of these is the following:

Definition 1.1. A space $X$ has a primitive base if and only if there is a sequence $\left\langle\mho_{n}: n \in N\right\rangle$ of well-ordered open covers of $X$ such that for all $p \in X$, the set $\left\{V\right.$ : for some $n, V$ is the first element of $\mho_{n}$ that contains $\left.p\right\}$ is a local base at $p$.

The purpose of this paper is to present a characterization that does not involve the notion of well ordering. This is done in terms of a function $h$ : $N \times X \rightarrow \tau$ (where $(X, \tau)$ is a space). Such functions have been used with notable success by Heath [3], [4] and Hodel [5] to characterize a number of interesting classes of spaces.

A simple condition and corresponding characterization theorem is first presented. Then some equivalent conditions are given. Applications to other types of spaces follow. Finally, it is pointed out, by example, that the condition given is not equivalent to Definition 1.1 when the axiom of choice $(=\mathrm{AC})$ is not assumed.

Presented to the Society, January 4, 1978; received by the editors January 4, 1978 and, in revised form, March 6, 1978.

AMS (MOS) subject classifications (1970). Primary 54E99; Secondary 54E30, 54A99.

Key words and phrases. Primitive base, base of countable order, $\theta$-space, first countable function, well ordering.

() American Mathematical Society 1978 
2. Initial functions. The main condition is defined here and the characterization theorem is proved.

Definition 2.1. Let $(X, \tau)$ be a space, and let $h: N \times X \rightarrow \tau$, and for all $p \in X$ and $n \in N, p \in h(n, p)$. The function $h$ is called initial if and only if for all $j \in N$ and $A \subseteq X$, if $A \neq \varnothing$, there exists $a \in A$ such that for all $b \in A$, either $h(j, a)=h(j, b)$ or $b \notin h(j, a)$. Such an element $a$ will be called a distinguished element of $A$.

The function $h$ is called first countable if and only if for all $p \in X,\{h(j, p)$ : $j \in N\}$ is a local base at $p$. It is called monotone if and only if $h(n, p) \supseteq h(n$ $+1, p)$ for all $n \in N$ and $p \in X$.

THeOREM 2.2. (AC) $A$ space has a primitive base if and only if it has a first countable initial function.

Proof. Suppose $X$ is a space with a primitive base. Then there is a sequence $\left\{\mathscr{W}_{n}: n \in N\right\}$ of well-ordered open covers of $X$ satisfying Definition 1.1. For each $j \in N$ and $p \in X$ let $h(j, p)$ be the first element of $\mathcal{Q}_{j}$ that contains $p$. Then $h: N \times X \rightarrow \tau$ is clearly a first countable function. If $j \in N, A \subseteq X$, and $A \neq \varnothing$, there is a first element $W$ of $\mho_{j}$ such that for some $p \in A, W=h(j, p)$. Let $a$ be such an element of $A$. If $b \in A$, then either $h(j, a)=h(j, b)$ or else $b \notin h(j, a)$. Thus $h$ is an initial function. Note that $\mathrm{AC}$ is not used here.

Suppose $X$ has an initial function $h$. Let $j \in N$. Suppose $X$ is well ordered and nonempty. Let $x_{0}$ denote the first distinguished element of $X$. Suppose $\lambda \in$ Ord and for all $\beta<\lambda, x_{\beta} \in X$ has been defined and is a distinguished element of $X_{\beta}=X \backslash \cup\left\{h\left(j, x_{\gamma}\right): \gamma<\beta\right\}$. If $X_{\lambda} \neq \varnothing$, let $x_{\lambda}$ be the first distinguished element of $X_{\lambda}$. If $X_{\lambda}=\varnothing$, the construction is finished. Thus there is some $\lambda \in$ Ord and a sequence $\left\langle x_{\alpha}: \alpha<\lambda\right\rangle$ such that for all $\alpha<\lambda, x_{\alpha}$ is a distinguished element of $X_{\alpha}$. If $\beta<\alpha<\lambda, x_{\alpha} \notin h\left(j, x_{\beta}\right)$. Thus the mapping $\alpha \mapsto h\left(j, x_{\alpha}\right)$ is one-to-one. Hence we may well order the set $\mathscr{W}_{j}=$ $\left\{h\left(j, x_{\alpha}\right): \alpha<\lambda\right\}$ by this mapping. By the definition of $\lambda, \mathscr{W}_{j}$ covers $X$. Let $F\left(x, \mathcal{O} \int_{j}\right)$ denote the first element of $\mathscr{W}_{j}$ that contains $x$. Then for some $\alpha<\lambda, h\left(j, x_{\alpha}\right)=F\left(x, \mathscr{W}_{j}\right)$. Since $x_{\alpha}$ is a distinguished element of $X_{\alpha}$, and $x \notin h\left(j, x_{\beta}\right)$ for $\beta<\alpha$, but $x \in h\left(j, x_{\alpha}\right)$, it follows that $h\left(j, x_{\alpha}\right)=h(j, x)$. Assuming that the above construction is carried out for each $j \in N$, it follows that $\left\langle\mathcal{W} \int_{n}: n \in N\right\rangle$ is a sequence of well-ordered open covers of $X$ and for all $p \in X$ and $n \in N, h(n, p)=F\left(p, \mathcal{W}_{n}\right)$ so that $\left\{F\left(p, \mathcal{Q} \int_{n}\right): n \in N\right\}$ is a local base at $p$.

In the proof of 2.2 , note that if $p \in X, h(n, p)=h\left(n, x_{\alpha}\right)$ for some $\alpha<\lambda$. Thus each $\mathcal{W}_{n}=\{h(n, p): p \in X\}$. We thus derive the following result.

Corollary 2.3. If $h$ is a monotone initial function on a space $X$, then $\langle\{h(n, p): p \in X\}: n \in N\rangle$ is an open primitive sequence (see [8] for definition) of $X$ in itself under the well ordering obtained in the proof of 2.2. 
3. A characterization of initiality. In this section initiality is decomposed into two other conditions. No topology really is involved, but the definitions are stated with respect to a topological space (as was 2.1) for brevity.

Definition 3.1. Let $(X, \tau)$ be a space and let $h: N \times X \rightarrow \tau$. The function $h$ is said to have a cycle if and only if for some $j \in N$ and $n \in N \backslash\{1\}$ there exist $x_{1}, \ldots, x_{n}$ such that

$$
h\left(j, x_{i}\right) \neq h\left(j, x_{k}\right) \text { for } 1 \leqslant i<k \leqslant n
$$

and $x_{i+1} \in h\left(j, x_{i}\right)$ for all $i<n$ and $x_{1} \in h\left(j, x_{n}\right)$. A function with no cycles is called acyclic. A function $h$ has an infinite descent if and only if for some $j \in N$ there is a sequence $\left\langle x_{n}: n \in N\right\rangle$ such that $h\left(j, x_{i}\right) \neq h\left(j, x_{k}\right)$ for all $i, k \in N$ with $i \neq k$, and $x_{i+1} \in h\left(j, x_{i}\right)$ for all $i \in N$. A function $h$ is called rooted if it has no infinite descents.

TheOREM 3.2. Let $(X, \tau)$ be a space. A function $h: N \times X \rightarrow \tau$ is initial if and only if it is rooted and acyclic.

Proof. Suppose $h$ is initial and $j \in N$. Suppose $\left\langle x_{n}: n \in N\right\rangle$ defines an infinite descent as in 3.1. Let $x_{m}$ be a distinguished element of $\left\{x_{n}: n \in N\right\}$. Then $x_{m+1} \notin h\left(j, x_{m}\right)$, a contradiction. Thus $h$ has no infinite descents. If $\left\langle x_{1}, \ldots, x_{n}\right\rangle$ defines a cycle relative to $j$, again let $x_{m}$ be a distinguished element of $\left\{x_{i}: 1 \leqslant i \leqslant n\right\}$. If $1 \leqslant i \leqslant n$ and $i \neq m, x_{i} \notin h\left(j, x_{m}\right)$, which leads to a contradiction. Thus $h$ is acyclic.

Suppose $h$ is rooted and acyclic. Suppose for some $j \in N, A \subseteq X, A \neq \varnothing$, and $A$ has no distinguished element. Let $x_{1} \in A$. Suppose $x_{1}, \ldots, x_{n}$ have been defined such that

$$
h\left(j, x_{i}\right) \neq h\left(j, x_{k}\right) \text { for } 1 \leqslant i<k \leqslant n
$$

and $x_{i+1} \in h\left(j, x_{i}\right)$ for $1 \leqslant i<n$. Since $x_{n}$ is not distinguished, there exists $x_{n+1} \in A$ such that $h\left(j, x_{n}\right) \neq h\left(j, x_{n+1}\right)$ and $x_{n+1} \in h\left(j, x_{n}\right)$. Suppose for some $k<n, h\left(j, x_{n+1}\right)=h\left(j, x_{k}\right)$. Then $x_{k} \in h\left(j, x_{n+1}\right)$ and $x_{m+1} \in$ $h\left(j, x_{m}\right)$ for $k \leqslant m \leqslant n$. This implies that $h$ has a cycle. Thus

$$
h\left(j, x_{n+1}\right) \neq h\left(j, x_{k}\right)
$$

for all $k \leqslant n$. Thus, by induction, a sequence defining an infinite descent of $h$ may be obtained. Thus $A$ must have a distinguished element.

4. Scattered decompositions. Another characterization of primitive base is given here.

Notation 4.1. If $f$ is a function on $N \times X, \mathcal{D}(n, f)$ denotes the decomposition determined by $x \mapsto f(n, x)$; i.e. if

$$
E(f(n, x))=\{y \in X: f(n, y)=f(n, x)\},
$$

then $\mathscr{D}(n, f)=\{E(f(n, x)): x \in X\}$.

DEFINITION 4.2. Let $(X, \tau)$ be a space and $f: N \times X \rightarrow \tau$. In the preceding notation, a decomposition $\mathscr{D}(n, f)$ is called scattered if and only if for every $\mathbb{Q} \subseteq \mathscr{D}(n, f)$, if $\mathbb{Q} \neq \varnothing$, there exists $M \in \mathbb{Q}$ and $U_{M} \in \tau$ such that $M \subseteq U_{M}$ 
and for all $K \in \mathbb{Q} \backslash\{M\}, U_{M} \cap K=\varnothing$. (This is a special instance of collectionwise scattered of [11].)

THEOREM 4.3. A space $(X, \tau)$ has a primitive base if and only if it has a first countable function $h: N \times X \rightarrow \tau$ such that all decompositions $\mathscr{D}(n, h)$ are scattered.

Proof. Suppose $X$ has a primitive base. By Theorem 2.2 it has a first countable initial function $h$. Let $n \in N$ and suppose $\varnothing \neq Q \in \mathcal{Q} D(n, h)$. Let $A=\{p \in X: E(h(n, p)) \in \mathbb{Q}\}$. Then $A$ has a distinguished element $a$. Let $M=E(h(n, a))$ and $U_{M}=h(n, a)$. Then $M \subseteq U_{M}$. Suppose $K \in \mathbb{Q} \backslash\{M\}$ and $b \in K$. Then $b \in A$ and $h(n, b) \neq h(n, a)$. Thus $b \notin U_{M}$.

Suppose $g$ is a first countable function with scattered decompositions. It may be seen (see [11, Theorem 2.3]) that each $\mathcal{D}(n, g)$ may be well ordered in such a way that for each $M \in \mathcal{Q}(n, g)$ there is an open $U_{M} \supseteq M$ such that $K \cap U_{M}=\varnothing$ for all $K$ following $M$. Define $h: N \times X \rightarrow \tau$ by

$$
h(n, x)=g(n, x) \cap U_{E(g(n, x))} \text {. }
$$

Then $h$ is first countable. Suppose $\varnothing \neq A \subseteq X$ and $n \in N$. Let $\mathbb{Q}=$ $\{E(g(n, x)): x \in A\}$ and let $M$ be the first element of $\mathcal{Q}$ in the well ordering on $\mathscr{D}(n, g)$. Then for some $a \in A, M=E(g(n, a))$. Suppose $b \in A$ and $h(n, a) \neq h(n, b)$. Then $g(n, a) \neq g(n, b)$, so $E(g(n, b))$ follows $M$. Thus $b \notin U_{M}$; hence $b \notin h(n, a)$. Therefore $h$ is initial.

5. Characterizations of base of countable order. The notion of initial function or functions with scattered decompositions may be used to characterize other kinds of spaces. As one example, we characterize bases of countable order. Recall that a set of interior condensation [6] in a space $(X, \tau)$ is a set $M$ for which there exists a sequence $\left\langle\mathcal{G}_{n}: n \in N\right\rangle$ of open sets such that if $G_{n+1} \subseteq G_{n} \in \mathcal{G}_{n}$ for all $n \in N$, then $\cap\left\{G_{n}: n \in N\right\} \subseteq M$, and for all $x \in M$ if $x \in G \in \mathcal{G}_{n}$, then there is $G^{\prime} \in \mathcal{G}_{n+1}$ such that $x \in G^{\prime} \subseteq G$.

THEOREM 5.1. A space is essentially $T_{1}$ and has a base of countable order if and only if it has a first countable function with scattered decompositions whose elements are sets of interior condensation.

Proof. Suppose $X$ is essentially $T_{1}$ and has a base of countable order. Then there exists an open primitive sequence $\left\langle\mathcal{H}_{n}: n \in N\right\rangle$ of $X$ (i.e. each $\mathcal{H}_{n}$ is a well-ordered (by $\leqslant$ ) open cover of $X$ such that for all $H \in \mathcal{H}_{n}$,

$$
p\left(H, \mathcal{H}_{n}\right)=H \backslash \cup\left\{H^{\prime} \in \mathscr{H}_{n}: H^{\prime}<H\right\} \neq \varnothing,
$$

and if $p \in X$,

$$
\left.F\left(x, \mathcal{H}_{n}\right)=\min \left\{H \in \mathcal{H}_{n}: x \in H\right\} \supseteq F\left(x, \mathcal{H}_{n+1}\right)\right)
$$

such that if $\left\langle H_{n}: n \in N\right\rangle$ is a sequence such that $H_{n} \in \mathcal{H}_{n}$ and $H_{n}=$ $F\left(H_{n+1}, \mathcal{H}_{n}\right)$ for all $n \in N$, then $\left\{H_{n}: n \in N\right\}$ is a base at all points of $\bigcap_{n \in N}\left\{H_{n}: n \in N\right\}$, [7]. Define $h(n, x)=F\left(x, \mathcal{H}_{n}\right)$ for all $n \in N$ and 
$x \in X$. Then $h$ is a first countable initial function. In a space having base of countable order, closed sets are sets of interior condensation. Since each $p\left(H, \mathcal{H}_{n}\right)$ is the intersection of a closed set and an open set, each $p\left(H, \mathcal{H}_{n}\right)$ is a set of interior condensation. In the notation of 4.1,

$$
p\left(H, \mathcal{H}_{n}\right)=E(h(n, x)) \text { if } H=F\left(x, \mathcal{H}_{n}\right) .
$$

Thus the condition is necessary.

Suppose $g$ is a function on $N \times X$ satisfying the stated condition. Then there is a sequence $\left\langle\mathcal{U}_{n}: n \in N\right\rangle$ of well-ordered open covers of $X$ and a function $h$ as in 4.3 such that $h(n, x)=F\left(x, \mathcal{Q} \int_{n}\right)$ for all $n \in N$ and $\{h(n, x): n \in N\}$ is a base at $x$. For each $n \in N$, define

$$
\mathcal{H}_{n}=\{h(1, x) \cap \cdots \cap h(n, x): x \in X\}
$$

for all $n \in N$ and well order $\mathcal{H}_{n}$ by the method of [9, Lemma 3.1] (an analogue of lexicographic order). Then one obtains an open primitive sequence $\left\langle\mathcal{H}_{n}: n \in N\right\rangle$ such that each $p\left(H, \mathcal{H}_{n}\right)$ is a set of interior condensation. By Theorem 4.2 of [9], $X$ is essentially $T_{1}$ and has base of countable order.

THeOREM 5.2. A space $(X, \tau)$ is essentially $T_{1}$ and has a base of countable order if and only if it has a first countable monotone initial function $h$ such that for all $p \in X$ and sequences $\left\langle x_{n}: n \in N\right\rangle$ and $\left\langle q_{n}: n \in N\right\rangle$ in $X$, if

$$
h\left(n, q_{n}\right)=h\left(n, q_{n+1}\right) \text { and }\left\{p, x_{n}\right\} \subseteq h\left(n, q_{n}\right)
$$

for all $n \in N$, then $\left\langle x_{n}: n \in N\right\rangle$ clusters at $p$.

Proof. If $X$ is essentially $T_{1}$ and has a base of countable order, it has an open primitive sequence $\left\langle\mathcal{H}_{n}: n \in N\right\rangle$ and a first countable monotone initial function $h$ defined by $h(n, x)=F\left(x, \mathcal{H}_{n}\right)$ as in the proof of 5.1. Suppose $h\left(n, q_{n}\right)=h\left(n, q_{n+1}\right)$. Then

$$
h\left(n, q_{n}\right)=F\left(h\left(n+1, q_{n+1}\right), \mathcal{H}_{n}\right) .
$$

Hence, if $\left\{p, x_{n}\right\} \subseteq h\left(n, q_{n}\right)$ for all $n,\left\{h\left(n, q_{n}\right): n \in N\right\}$ is a base at $p$ and $\left\langle x_{n}: n \in N\right\rangle$ clusters at $p$.

Suppose $h$ is a function on $N \times X$ to $\tau$ as described. For each $n \in N$, let $\mathcal{G}_{n}=\{U \in \tau$ : for some $x \in U, U \subseteq h(n, x)\}$. Each $\mathcal{G}_{n}$ is a base for $X$. Suppose $G_{n+1} \subseteq G_{n} \in \mathcal{G}_{n}$ and $p \in G_{n}$ for all $n \in N$. For each $n \in N, G_{n} \subseteq$ $h(n, x)$ for some $x \in X$. Thus the set $A_{n}=\left\{x \in X\right.$ : for some $j \in N, G_{j} \subseteq$ $h(n, x)\} \neq \varnothing$. Thus there is a distinguished element $q_{n} \in A_{n}$. Suppose $n \in N$. There exists $j>n+1$ such that $G_{j} \subseteq h\left(n, q_{n}\right) \cap h\left(n+1, q_{n+1}\right)$. Also there is $x_{j} \in G_{j}$ such that $G_{j} \subseteq h\left(j, x_{j}\right)$. Hence $G_{j} \subseteq h\left(n, x_{j}\right)$, by monotonicity, so $x_{j} \in A_{n}$. Since $x_{j} \in h\left(n, q_{n}\right)$, it follows that $h\left(n, q_{n}\right)=h\left(n, x_{j}\right)$. Similarly $h\left(n+1, q_{n+1}\right)=h\left(n+1, x_{j}\right)$. Hence $q_{n+1} \in h\left(n, q_{n}\right)$. Since $G_{j} \subseteq h\left(n, q_{n+1}\right)$, $q_{n+1} \in A_{n}$. Thus $h\left(n, q_{n}\right)=h\left(n, q_{n+1}\right)$. If $U$ is open and $p \in U$, but no $h\left(n, q_{n}\right) \subseteq U$, there exists $x_{n} \in h\left(n, q_{n}\right) \backslash U$ for each $n \in N$. By hypothesis, $\left\langle x_{n}: n \in N\right\rangle$ clusters at $p$, so $p \notin U$, a contradiction. Thus $\left\{h\left(n, q_{n}\right): n \in N\right\}$ 
is a base at $p$ and, thus, so is $\left\{G_{n}: n \in N\right\}$.

6. Initiality versus well ordering. From the proof of 2.2 it may be seen that having a primitive base implies the existence of an initial function. To go from initial function to primitive base requires the axiom of choice. The following two theorems relate to this.

THEOREM 6.1. A discrete space has a primitive base if and only if it can be well ordered.

Proof. The sufficiency is clear. Suppose $X$ is discrete and has a primitive base defined by a sequence $\left\langle\mathcal{W}_{n}: n \in N\right\rangle$ as in 1.1. Since for each $p \in X$, $\left\{F\left(p, W_{n}\right): n \in N\right\}$ is a base at $p$, there exists an integer $n$ such that $F\left(p, \mathcal{W}_{n}\right)=\{p\}$. For each $p \in X$, let $n(p)$ denote the first such integer. For each $n \in N,\{p: n(p)=n\}$ can be well ordered by taking $p<q$ if and only if $\{p\}<\{q\}$ in $\mho_{n}$. Hence $X$ can be written as the well-ordered union of well-ordered sets.

THEOREM 6.2. If AC does not hold, having a first countable initial function need not imply primitive base.

Proof. Suppose $X$ is a set (in some consistent model of $Z F$ ) which cannot be well ordered. Then if $\tau$ is the discrete topology on $X,(X, \tau)$ does not have a primitive base, by Theorem 6.1. The function $h: N \times X \rightarrow \tau$ defined by $h(j, p)=\{p\}$ for all $j \in N$ and $p \in X$ is first countable and initial.

Thus without AC, not all metric spaces have primitive bases. The following theorem holds without AC.

THEOREM 6.3. If $(X, d)$ is a metric space with a well-ordered dense subset, then $(X, d)$ has a primitive base.

Proof. Let $B(x, \varepsilon)=\{y \in X: d(x, y)<\varepsilon\}$. For each $n \in N$ let $\mathscr{W}_{n}=$ $\{B(z, 1 / n): z \in D\}$. For each $W \in \mathcal{Q W}_{n}$, let $z_{W}=\min \{z: W=B(z, 1 / n)\}$. Then $W \mapsto z_{W}$ is a one-to-one map of $\mathscr{W}_{n}$ into $D$. Hence $\mathcal{W}_{n}$ may be well ordered by the inverse of this map. If $x \in \cap\left\{B\left(z_{n}, 1 / n\right): n \in N\right\}$, then $\left\{B\left(z_{n}, 1 / n\right): n \in N\right\}$ is a base at $x$.

COMmENTS 6.4. The Sorgenfrey line $S$ does not have a primitive base (since a regular hereditary Lindelöf space having a primitive base is metrizable [10]). Hence it has no first countable initial function. For $n \in N$ and $x \in S$, let $g(n, x)=[x, x+1 / n[$. Then $g$ is a first countable acyclic $\theta$-function. For $g$ is first countable and $x \in g(n, y)$ and $y \in g(n, x)$ implies $x=y$. Thus $g$ is not rooted. In fact, if $j \in N$ and $x_{i}=-1 /(j+i), x_{i+1} \in g\left(j, x_{i}\right)$ for all $i \in N$ and $g\left(j, x_{i}\right) \neq g\left(j, x_{k}\right)$ for $i \neq k$.

6.5. An example of a rooted first countable function that is not acyclic may easily be constructed in $[0,1]$.

6.6. A reading of [10], where it is pointed out that primitive base implies $\theta$-space, provided motivation for a functional approach to primitive base. 
6.7. The idea of initial function may be fruitfully used in non first countable situations. Details of this will appear elsewhere.

\section{REFERENCES}

1. D. K. Burke and D. J. Lutzer, Recent advances in the theory of generalized metric spaces, Topology, Proc. of the Memphis State University Conference, Marcel Dekker, New York, 1976, pp. 1-70. MR 55 \# 1318.

2. P. Fletcher and W. F. Lindgren, $\theta$-spaces, General Topology and Appl. (to appear).

3. R. Heath, Arcwise connectedness in semi-metric spaces, Pacific J. Math. 12 (1965), 91-96.

4. On open mappings and certain spaces satisfying the first countability axiom, Fund. Math. 62 (1965), 91-96.

5. R. Hodel, Spaces defined by sequences of open covers which guarantee that certain sequences have cluster points, Duke Math. J. 39 (1972), 253-263.

6. H. H. Wicke, Open continuous images of certain kinds of $M$-spaces and completeness of mappings and spaces, General Topology and Appl. 1 (1971), 85-100.

7. H. H. Wicke and J. M. Worrell, Jr., Primitive structures in general topology, Studies in topology, Academic Press, New York, 1975, pp. 581-599. MR 51 \# 11438.

8. __ A characterization of primitive bases, Proc. Amer. Math. Soc. 50 (1975), 443-450.

9. A characterization of spaces having bases of countable order in terms of primitive bases, Canad. J. Math. 27 (1975), 1100-1109.

10. _ The hereditary Lindelö property, primitive structures, and separable metrizability, Notices Amer. Math. Soc. 22 (1975), A-425, Abstract 723-G10.

11. On spaces which are scattered with respect to collections of sets, Topology Proceedings 2 (1977), 281-307.

Department of Mathematics, Ohio University, Athens, Oho 45701 